\title{
Sirolimus-Eluting Stent for In-Stent Restenosis of Left Main Coronary Artery in Takayasu Arteritis
}

\author{
Yutaka Furukawa, MD; Toshihiro Tamura, MD; Masanao Toma, MD; Mitsuru Abe, MD; \\ Naritatsu Saito, MD; Natsuhiko Ehara, MD; Ryoji Taniguchi, MD; \\ Yoshihisa Nakagawa, MD; Toru Kita, MD; Takeshi Kimura, MD
}

\begin{abstract}
A 53-year-old woman with Takayasu arteritis was admitted to hospital because of worsening exertional angina. Coronary angiography revealed $90 \%$ ostial stenosis in the left main coronary artery (LMCA), which also involved the bifurcation of the relatively short LMCA. Because the patient refused coronary bypass surgery, she underwent percutaneous coronary intervention (PCI) and the stenosis was successfully dilated. However, the exertional angina recurred a few months later and again after the second PCI. Finally, a sirolimus-eluting stent was deployed in the in-stent restenotic lesion. The patient has been free from angina pectoris for 6 months after the last PCI and follow-up coronary angiography indicated no restenosis in the LMCA. (Circ J 2005; 69: 752-755)
\end{abstract}

Key Words: Left main coronary artery disease; Restenosis; Sirolimus-eluting stent; Takayasu arteritis

$\mathbf{T}$ akayasu arteritis is a form of large vessel vasculitis with an unclear pathogenesis, although autoimmune mechanisms as well as a genetic etiology are possibly involved in its evolution and progression. Because Takayasu arteritis affects the entire aorta and its major branches, it occasionally complicates coronary artery stenoses. Although revascularization therapy is recommended for such patients, obstructive lesions in the subclavian arteries, active inflammation or severe calcification of the aorta often render coronary bypass grafting impossible. In addition, the long-term outcome of percutaneous coronary intervention (PCI) in patients with Takayasu arteritis is largely unknown. We describe a case of Takayasu arteritis with left main coronary artery (LMCA) stenosis that was successfully treated by sirolimus-eluting stent (SES) deployment after repeated in-stent restenosis following bare metal stenting.

\section{Case Report}

A 53-year-old woman was admitted to hospital because of worsening exertional angina. At the age of 42 years, she had been diagnosed as having Takayasu arteritis. Magnetic resonance angiography and $99 \mathrm{~m}$ technetium pulmonary perfusion scintigraphy revealed an occlusion of the left pulmonary artery and multiple mild stenoses in both of the common carotid and subclavian arteries. She had been treated with oral glucocorticoids (prednisolone) and was generally in good condition for the past 11 years until she presented with exertional angina. Angiography in August 2003 revealed severe (90\%) ostial stenosis in a short

(Received September 6, 2004; accepted October 13, 2004) Department of Cardiovascular Medicine, Kyoto University Graduate School of Medicine, Kyoto, Japan

Mailing address: Yutaka Furukawa, MD, Department of Cardiovascular Medicine, Kyoto University Graduate School of Medicine, 54 Kawaharacho, Shogoin, Sakyo-ku, Kyoto 606-8397, Japan. E-mail: yutakaf@kuhp.kyoto-u.ac.jp
LMCA with involvement of the distal bifurcation (Fig 1A), an intact right coronary artery (Fig 1B) and total occlusion of the left pulmonary artery (Fig 1C). Collateral circulation via the intercostal arteries had developed between the left internal thoracic artery and left pulmonary circulation. Although coronary bypass surgery was recommended as the first therapeutic option, the patient refused open-chest surgery and PCI was then considered as another option. Because the patient developed unstable angina, a bare metal stent (3.0 $\times 15 \mathrm{~mm}$ ACS RX Multi-Link ${ }^{\mathrm{TM}}$, Guidant) was urgently implanted in her LMCA in September 2003; at that time, SES were not available in Japan. The kissing balloon technique was used for stenosis of the bifurcation of the left anterior descending artery (LAD) $(4.0 \times 15 \mathrm{~mm}$ POWERSAIL, Guidant) and left circumflex artery (LCX) $(2.5 \times 20 \mathrm{~mm}$ Maverick2, Boston Scientific). The 90\% LMCA stenosis was successfully dilated to $0 \%$ (Fig 1D) and the angina completely disappeared. The daily dose of oral prednisolone was increased from $12.5 \mathrm{mg}$ to $20 \mathrm{mg}$ because the blood test suggested enhanced activity of the Takayasu arteritis, showing positive C-reactive protein (CRP: $3.6 \mathrm{mg} / \mathrm{dl}$ ) and an augmented erythrocyte sedimentation rate $(48 \mathrm{~mm} / \mathrm{h})$. These inflammatory parameters normalized within 1 month. However, 3 months later, the patient was readmitted for recurrence of the angina. She underwent balloon angioplasty using a $4.0 \times 18 \mathrm{~mm}$ POWERSAIL (Guidant) for the LMCA and LAD, and $2.5 \times 15 \mathrm{~mm}$ Stormer (Medtronic) for the LCX and the restenosed LMCA lesion was successfully treated. Two months after the second PCI, the patient underwent a third PCI for $90 \%$ in-stent restenosis in the LMCA because the angina had recurred. Because of the rapid progression of the repeated restenosis accompanied by extension of the stenosis to ostial LAD and LCX, a SES $(3.5 \times 23 \mathrm{~mm}$ Cypher, Johnson \& Johnson) was implanted for the LMCA and LAD in-stent restenosis lesion. The kissing balloon technique was used with a $4.5 \times 20 \mathrm{~mm}$ Quantum Maverick (Boston Scientific) for the LMCA and LAD, and 2.75× 

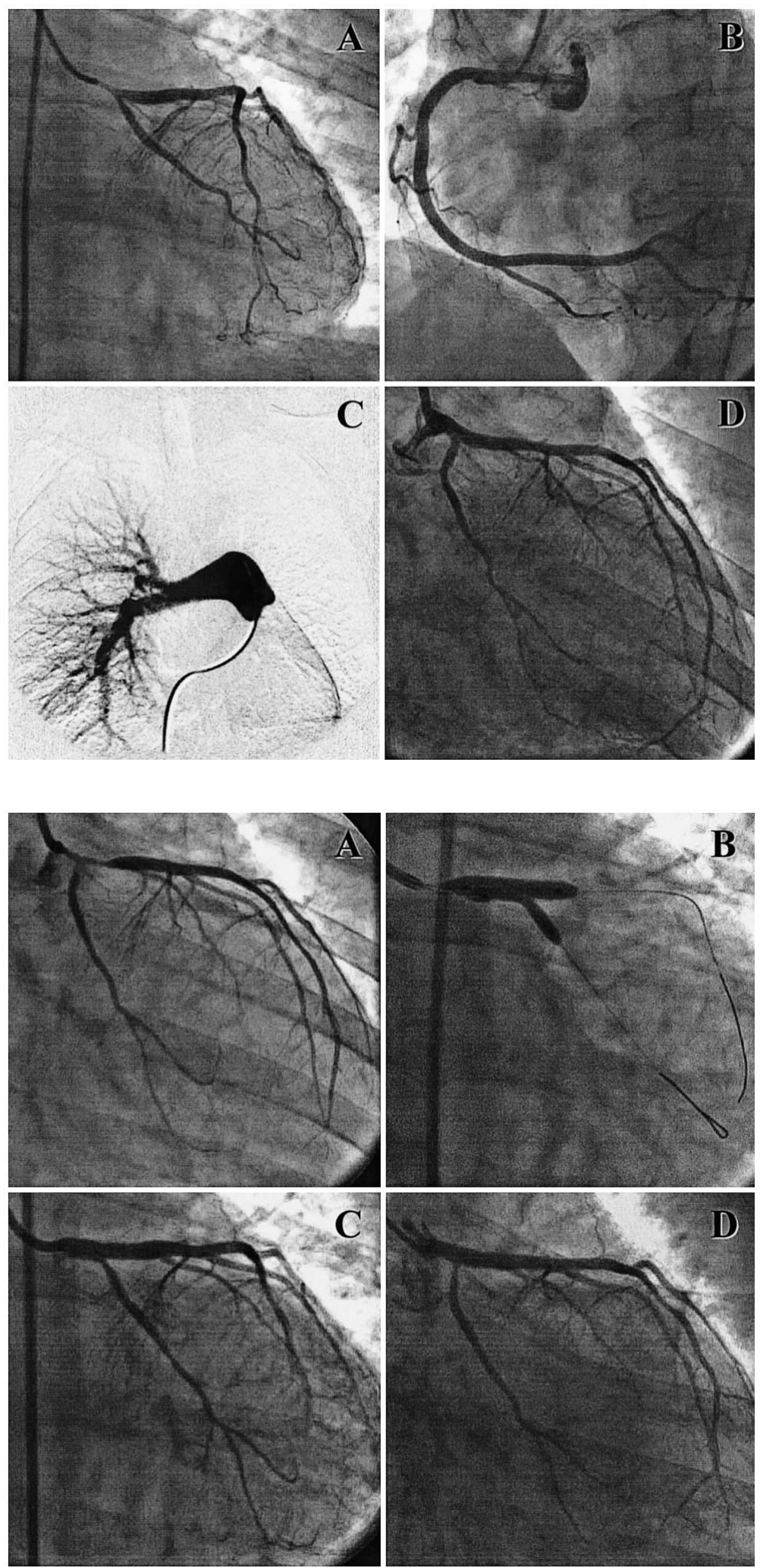

Fig 1. Coronary and pulmonary angiographic findings during the first hospitalization for angina. (A) Left coronary selective injection revealed $90 \%$ stenosis in the ostium of the left main coronary artery (LMCA). (B) Intact right coronary artery. (C) Totally occluded left pulmonary artery. (D) Left coronary angiography after percutaneous coronary stenting. The $90 \%$ stenosis of the LMCA was successfully dilated to $0 \%$.

Fig 2. Coronary angiographic findings during the sirolimus-eluting stent implantation $(\mathrm{A}-\mathrm{C})$ and at 6month follow-up. (A) Left coronary angiography shows the $90 \%$ in-stent restenosis in the left main coronary artery (LMCA) and ostial left anterior descending artery (LAD). The lesion also involved the ostium of the left circumflex artery (LCX: 90\% stenosis). (B) Sirolimus-eluting stent deployed in the LMCA and ostial LAD using the kissing balloon technique (see text for details). (C) The succcessfully dilated lesion: $0 \%$ for the $\mathrm{LMCA}$ and $\mathrm{LAD}, 50 \%$ for the LCX. (D) At 6-month follow-up, selective left coronary injection shows no significant restenosis.
$20 \mathrm{~mm}$ Maverick2 for the LCX (Fig 2A-C).

After the third PCI using the SES, the patient has been free from angina pectoris and follow-up coronary angiography 6 months later showed only minimal luminal narrowing in the LMCA and ostial LCX (Fig 2D).

\section{Discussion}

Takayasu arteritis is a large vessel vasculitis that involves the aorta and its main branches, the pulmonary arteries and the coronary arterial tree. However, angina pectoris because of the coronary involvement is not a common feature, having been reported in 6-19\% of cases. ${ }^{1-4}$ Most of the 
coronary stenoses are ostial stenoses of the LMCA and/or right coronary artery.,5 Coronary revascularization surgery in patients with Takayasu arteritis is often problematic. Stenoses or occlusions of the innominate and subclavian arteries reduce the availability of the internal thoracic arteries as coronary bypass grafts, and active inflammation in the aorta may require an arterial patch with vein grafts at the aortic anastomoses6 Moreover, Takayasu arteritis is frequently complicated by severe calcification in the aorta, which may also make the bypass surgery arduous? For such patients, PCI could be a more beneficial coronary revascularization strategy than coronary bypass grafting.

Recently, PCI has been used as the revascularization therapy for unprotected atherosclerotic LMCA stenosis in poor surgical candidates. Although it has been regarded as contraindicated because of its unacceptably high mortality rate and coronary bypass surgery has been the preferred option, advances in techniques and equipment have enabled stenting for unprotected LMCA to be performed with a high initial success rate and acceptable short- to intermediate-term outcome? Favorable long-term results of stenting have been also reported in selected patients with normal left ventricular function, although restenosis occurred in approximately $20 \%$ of the patients and many of the restenosed lesions required revascularization therapy. In the era of bare metal stenting, restenosis has been the leading cause of major adverse cardiac events after LMCA stenting and has restricted its indication? Predictors of refractory restenosis include a short time to restenosis.11 The present case had rapid progression of repeated restenosis and the stenosis extended during each progression. Thus, this is definitely a case of refractory restenosis with a rather poor prognosis. By the time of the third PCI, the distal bifurcation of LMCA was totally involved in the restenotic lesion. PCI for an unprotected LMCA bifurcation lesion is still technically difficult and remains a challenge. Although a variety of strategies have been attempted, residual stenosis in the side-branch ostium and a high rate of restenosis are still common problems $!^{12}$ In the present case, there was still 50\% stenosis of the side branch (LCX) ostium after the third PCI in which a SES was deployed in the parent vessel (LMCA to LAD) and significant late restenosis in the LCX ostium was predicted. However, follow-up angiography at 6 months indicated only minimal additional narrowing of the LCX ostium and 1 possible explanation is the antirestenotic effects of the eluted sirolimus. Colombo et al have recently reported a lower incidence of restenosis in bifurcation coronary lesions treated with SES and using the kissing balloon technique than in historical bare metal stent controls; nevertheless, restenosis of the side branch remains a problem. ${ }^{13}$ An aorto-coronary ostial lesion, which was another characteristic of the present case, is also prone to restenosis, but significantly reduced incidence of restenosis after SES deployment has been reported for such lesions! 14 Thus, SES shows promise in reducing the rate of restenosis in bifurcation and ostial lesions.

Because most studies of coronary stenting have involved subjects with coronary stenoses of common atherosclerotic etiology, it is uncertain whether coronary stenosis with a prominent inflammatory/immune-mediated etiology such as Takayasu arteritis can be treated as safely and effectively by stenting. Although the results of coronary stenting for Takayasu arteritis have not been reported even in a small group of patients, favorable results of percutaneous stent implantation for aortic as well as major branch stenoses have been indicated 15 In addition, the rate of restenosis after percutaneous transluminal renal angioplasty (PTRA) in chronic stable Takayasu arteritis appears to be lower than for PTRA in common atherosclerotic lesions ${ }^{16}$

The background activity of inflammatory diseases such as Takayasu arteritis may also affects the initial and longterm results of PCI. Arterial stenotic lesions often progress very rapidly in Takayasu arteritis when it is in an active inflammatory phase ${ }^{17}$ In the present case, the serum CRP concentration and erythrocyte sedimentation rate were increased at the occurrence of angina, suggesting the recurrence of arterial inflammation caused by the Takayasu arteritis. Although these parameters gradually improved with an increased dosage of prednisolone, enhanced arterial inflammation might have played an important role in the refractory rapid progression of the restenosis after coronary stenting, as well as in the initial onset of angina and its worsening.

Pathological examinations of cases of Takayasu arteritis have shown predominant intimal and adventitial infiltration of T lymphocytes colocalizing with dendritic cells. Dense fibrosis replaces the destroyed medial structure, which normally consists of smooth muscle cells and layered elastic fibers ${ }^{18}$ Expression of major histocompatibility complex (MHC)-I, MHC-II and co-stimulatory molecules on the infiltrating cells has also been reported 19,20 These observations suggest that vascular inflammation driven by antigen-specific immune reactions plays a key role in the pathogenesis of the arterial stenoses in Takayasu arteritis and may also facilitate restenosis after coronary stenting. Sirolimus was originally discovered as an antibiotic and then developed as an immunosuppressive agent. It inhibits cell-cycle progression at the late $\mathrm{G}_{1}$ stage and suppresses activated T cell proliferation ${ }^{1}{ }^{1}$ In addition to its inhibitory effects on $\mathrm{T}$ cells, sirolimus markedly suppress the allostimulatory activity of antigen-presenting dendritic cells? 22 These immunosuppressive properties of sirolimus may have a beneficial synergistic effect with its anti-proliferative action on vascular smooth muscle cells in the prevention of restenosis after coronary stenting in Takayasu arteritis.

In conclusion, a case of Takayasu arteritis with LMCA in-stent restenosis was successfully treated by a SES. Because of its immunosuppressive effects in the inflamed arterial walls, the SES shows promise for the treatment of stenotic lesions in patients with Takayasu arteritis.

\section{References}

1. Koide K. Takayasu arteritis in Japan. Heart Vessels Suppl 1992; 7: $48-54$.

2. Hall S, Barr W, Lie JT, Stanson AW, Kazmier FJ, Hunder GG. Takayasu arteritis: A study of 32 North American patients. Medicine (Baltimore) 1985; 64: 89-99.

3. Cipriano PR, Silverman JF, Perlroth MG, Griepp RB, Wexler L. Coronary arterial narrowing in Takayasu's aortitis. Am J Cardiol 1977; 39: 744-750.

4. Endo M, Tomizawa Y, Nishida H, Aomi S, Nakazawa M, Tsurumi $\mathrm{Y}$, et al. Angiographic findings and surgical treatments of coronary artery involvement in Takayasu arteritis. J Thorac Cardiovasc Surg 2003; 125: 570-577.

5. Matsubara O, Kuwata T, Nemoto T, Kasuga T, Numano F. Coronary artery lesions in Takayasu arteritis: Pathological considerations. Heart Vessels Suppl 1992; 7: 26-31.

6. Nakano S, Shimazaki Y, Kaneko M, Taniguchi K, Miyamoto Y, Takami H, et al. Transaortic patch angioplasty for left coronary ostial stenosis in a patient with Takayasu's aortitis. Ann Thorac Surg 1992; 53: 694-696.

7. Nishiyama A, Matsubara S, Toyama J. Takayasu arteritis with multi- 
ple cardiovascular complications. Heart Vessels 2001; 16: 23-27.

8. Kelley MP, Klugherz BD, Hashemi SM, Meneveau NF, Johnston $\mathrm{JM}$, Matthai WH Jr, et al. One-year clinical outcomes of protected and unprotected left main coronary artery stenting. Eur Heart $J$ 2003; 24: $1554-1559$.

9. Black A, Cortina R, Bossi I, Choussat R, Fajadet J, Marco J. Unprotected left main coronary artery stenting: Correlates of midterm survival and impact of patient selection. J Am Coll Cardiol 2001; 37: $832-838$.

10. Park SJ, Park SW, Hong MK, Lee CW, Lee JH, Kim JJ, et al. Longterm (three-year) outcomes after stenting of unprotected left main coronary artery stenosis in patients with normal left ventricular function. Am J Cardiol 2003; 91: 12-16.

11. Alfonso F, Cequier A, Zueco J, Moris C, Suarez CP, Colman T, et al. Stenting the stent: Initial results and long-term clinical and angiographic outcome of coronary stenting for patients with in-stent restenosis. Am J Cardiol 2000; 85: 327-332.

12. Sheiban I, Albiero R, Marsico F, Dharmadhikari A, Tzifos V, Pagnotta P, et al. Immediate and long-term results of "T" stenting for bifurcation coronary lesions. Am J Cardiol 2000; 85: 1141-1144, A9.

13. Colombo A, Moses JW, Morice MC, Ludwig J, Holmes DR Jr, Spanos V, et al. Randomized study to evaluate sirolimus-eluting stents implanted at coronary bifurcation lesions. Circulation 2004; 109: $1244-1249$.

14. Iakovou I, Ge L, Michev I, Sangiorgi GM, Montorfano M, Airoldi F, et al. Clinical and angiographic outcome after sirolimus-eluting stent implantation in aorto-ostial lesions. J Am Coll Cardiol 2004; 44:
967-971.

15. Nomura M, Kida S, Yamashima T, Yamashita J, Yoshikawa J, Matsui O. Percutaneous transluminal angioplasty and stent placement for subclavian and brachiocephalic artery stenosis in aortitis syndrome. Cardiovasc Intervent Radiol 1999; 22: 427-432.

16. Chatterjee SS, Pahari DK, Sharma RK, Halder B, Roy S, Ghosh S, et al. Long term follow-up of percutaneous transluminal renal angioplasty with special reference to aorto-arteritis. Indian Heart J 1995; 47: $120-124$.

17. Numano F, Ohta N, Sasazuki T. HLA and clinical manifestations in Takayasu disease. Jpn Circ J 1982; 46: 184-189.

18. Inder SJ, Bobryshev YV, Cherian SM, Lord RS, Masuda K, Yutani C. Accumulation of lymphocytes, dendritic cells, and granulocytes in the aortic wall affected by Takayasu's disease. Angiology 2000; 51: $565-579$.

19. Seko Y. Takayasu arteritis: Insights into immunopathology. Jpn Heart J 2000; 41: 15-26.

20. Seko Y, Sugishita K, Sato O, Takagi A, Tada Y, Matsuo H, et al. Expression of costimulatory molecules (4-1BBL and Fas) and major histocompatibility class I chain-related A (MICA) in aortic tissue with Takayasu's arteritis. $J$ Vasc Res 2004; 41: 84-90.

21. Flanagan WM, Crabtree GR. Rapamycin inhibits p34cdc2 expression and arrests T lymphocyte proliferation at the G1/S transition. Ann NY Acad Sci 1993; 696: 31-37.

22. Chiang PH, Wang L, Bonham CA, Liang X, Fung JJ, Lu L, et al. Mechanistic insights into impaired dendritic cell function by rapamycin: Inhibition of Jak2/Stat4 signaling pathway. J Immunol 2004; 172: $1355-1363$. 